This item was submitted to Loughborough's Research Repository by the author.

Items in Figshare are protected by copyright, with all rights reserved, unless otherwise indicated.

\title{
Examining the influence of expertise on the effectiveness of diagramming and summarising when studying scientific materials
}

PLEASE CITE THE PUBLISHED VERSION

https://doi.org/10.1080/03055698.2017.1390444

\section{PUBLISHER}

Informa UK Limited, trading as Taylor \& Francis Group

VERSION

AM (Accepted Manuscript)

\section{PUBLISHER STATEMENT}

This is an Accepted Manuscript of an article published by Taylor \& Francis in Educational Studies on 17 October 2017, available online: http://www.tandfonline.com/10.1080/03055698.2017.1390444.

\section{LICENCE}

CC BY-NC-ND 4.0

\section{REPOSITORY RECORD}

Chen, Ouhao, Emmanuel Manalo, and Yan She. 2017. "Examining the Influence of Expertise on the Effectiveness of Diagramming and Summarising When Studying Scientific Materials". figshare. https://hdl.handle.net/2134/12052665.v1. 
Examining the Influence of Expertise on the Effectiveness of Diagramming and

\title{
Summarizing When Studying Scientific Materials
}

\begin{abstract}
A 2 (learning strategies: diagram vs. summary) x 2 (levels of expertise: low vs. high) experiment was conducted to compare the effectiveness of using diagrams to writing summaries for students given biological information to learn and who possessed different levels of expertise in that topic area. A main effect of learning strategy used on number of idea units encoded (in diagrams or summaries) was found: drawing diagrams was superior to writing summaries. However, no interaction effect between learning strategies and expertise was found. An examination of students' subjective ratings of cognitive load revealed that those with low expertise reported higher levels of cognitive load when constructing diagrams. These findings suggest that using diagrams is effective for identifying and encoding important information when learning, but that it would be helpful to provide guidance about diagram use particularly to students who are novices in the topic area to reduce cognitive load.
\end{abstract}

Keywords: learning strategy use, diagramming, summarizing, cognitive load, expertise, science learning 


\section{Introduction}

Constructing external representations, such as diagrams, is considered an effective way for facilitating more successful learning, problem solving, and communication (e.g., Ainsworth, Prain, \& Tytler, 2011; Butcher, 2006). However, learner-related factors, such as expertise in the topic being dealt with and cognitive load associated with diagram construction, may affect students' predisposition toward diagram use. In this paper, these possible influences are examined to better understand how it may be possible to promote effective diagram use among students to benefit their educational achievement. Note that the primary focus of this research is on the effects of drawing diagrams; summarizing was used only as a strategy to compare with diagramming. Thus, although some pertinent research about summary writing is referred to, the majority of research that is reviewed and discussed focuses on diagram construction and use.

\section{The Effectiveness - and Problems - of Using Diagrams in Learning Situations}

Many studies have investigated the effectiveness of using diagrams in various learning situations, and most of them have revealed favorable outcomes as a consequence of such use (Leopold, Doerner, Leutner, \& Dutke, 2015; Leopold \& Leutner, 2012; Uesaka, Manalo, \& Ichikawa, 2007; Van Meter, 2001). For example, Leopold and Leutner (2012) compared the effectiveness of generating diagrams with summarizing and selecting main ideas in learning science texts. They reported that diagram generation was more effective in terms of both comprehension and 
subsequent transfer, which they attributed to diagram construction providing a visual-spatial component to the information being learned, and thus facilitating use of both verbal and visual channels of working memory. However, Leopold and Leutner did not examine the relative amounts of information that participants encoded. If more information gets encoded when diagramming, this may provide an additional explanation for why diagram use results in more successful learning outcomes.

There are, however, studies that have identified some problems and limitations associated with diagram use (e.g., De Bock, Verschaffel, Janssens, Van Dooren, \& Claes; 2003; Hegarty \& Kozhevnikov, 1999). Hegarty and Kozhevnikov (1999), for example, compared generation of schema-based representations with generation of picture-based representations in math word problem solving, and found that students' schematic representations were positively related with successful solutions, while their pictorial representations were not. Their results suggest that for diagrams to be helpful in problem solving, they need to represent important relationships between objects of the problem rather than irrelevant pictorial details.

Another important problem concerning diagram use in learning contexts is the lack of spontaneity with which students use them. In other words, despite learning about diagrams and being shown by their teachers how to use diagrams, the majority of students tend not to use diagrams of their own volition. This problem has been reported in both the areas of math problem solving and written communication (e.g., Dufour-Janvier, Bednarz, \& Belanger, 1987; Manalo \& Uesaka, 2012; Manalo, Uesaka, Pérez-Kriz, Kato, \& Fukaya, 2013; Uesaka et al., 2007). However, the ability 
to effectively use diagrams is considered an essential skill in 21st Century environments (e.g., Pellegrino \& Hilton, 2012). Thus, understanding and seeking solutions to this problem is an important challenge in education (Manalo \& Uesaka, 2016; Uesaka, Manalo, \& Ichikawa, 2010).

\section{Cognitive Load, Expertise, and Drawing Diagrams}

There are two main types of cognitive load, intrinsic load and extraneous load (Kalyuga, 2011). Intrinsic load is determined by the nature of information that learners must deal with (Sweller, 1994); it arises from the interaction between the nature of the information and the expertise of the learner (Van Merriënboer, Kester, \& Paas, 2006) and is therefore directly relevant to learning. Extraneous cognitive load, on the other hand, is load that is imposed by the way the information is presented: it is not necessary for learning and can be altered by instructional interventions (Sweller, Van Merrienboer, \& Paas, 1998). In fact, this load should be reduced or even eliminated (Kalyuga, 2011) to make more working memory resources available for dealing with intrinsic load, and thus improve learning.

Leutner, Leopold, and Sumfleth (2009) investigated the issue of how drawing diagrams might affect cognitive load. In one of the conditions in their study, they asked students to draw a picture of information in scientific text they were given to read. Their results indicated that drawing increased cognitive load and reduced text comprehension. Schwamborn, Thillmann, Opfermann, and Leutner (2011) obtained similar results. They found that, while presenting pictures to participants reduced cognitive load and enhanced information retention in learning, generating pictures 
increased cognitive load and reduced information retention.

The negative learning outcomes obtained for diagram generation in these two studies are understandable when the nature of the information they asked participants to draw (abstract, hard to imagine) is taken into account. Manalo and Uesaka (2012, 2014) explained that hard-to-imagine information demanded high amounts of cognitive resources to generate diagrams for, and attempts at generating diagrams for such information deplete working memory of resources that could be used in other concurrently undertaken learning-related tasks. These findings suggest that it would be important to investigate the effect of learner expertise on both cognitive load and the usefulness of strategies like diagram generation as, presumably, expertise would influence cognitive load and the resulting availability of cognitive resources for learning.

The present authors have found very few studies that have investigated the relationship between subject/topic expertise and diagram construction in learning contexts. A few studies by Grawemeyer and Cox (Cox \& Grawemeyer, 2003; Grawemeyer \& Cox, 2004, 2008) have examined the effects of participant knowledge on diagram selection and sorting, but they did not require participants to actually generate diagrams. Anzai (1991) did require a student with no knowledge about physics (and therefore a novice in the subject) to study a chapter in a physics textbook, and examined the notes the student produced - including the diagrams she constructed. This examination revealed limitations and errors, as well as some progress over time in the diagram drawing and inference capabilities of the student - but as noted, this 
study was based only on the performance of one participant.

\section{Expertise, Motivation, and Learning}

Regarding the relationship between expertise and learning performance, one would normally assume that expertise in some topic area would lead to better learning performance in that topic area because knowledge structures present in the long-term memory of experts would perform organizing and facilitating roles in the execution of complex cognitive processes involved in learning (e.g., Kalguya \& Sweller, 2005;

Sweller, 2003). However the findings of some studies suggest that expertise can also have a detrimental effect on learning performance. The findings of Wood and Lynch (2002), for example, revealed that people who possess prior knowledge about information they have to learn tend to get complacent and not pay enough attention during encoding; they therefore end up learning less compared to people who start off knowing nothing at all about the material to be learned. Therefore, although expertise can equip people with the cognitive structures to facilitate better learning outcomes, possession of expertise (i.e., in the form of prior knowledge) can also lead to complacency, leading to subsequent poor outcomes in learning.

There are also various environmental conditions that could affect the learning motivation of school students, which in turn could affect how much and how well they learn. One such condition is whether the material to be learned will be examined or not. Especially in the Chinese school context, whether something will be examined significantly affects students' motivation to learn it, as it is in exams that "parents 
hope their children will excel" (Chen, Warden, \& Chang, 2005, p. 625). The potential influence of this particular condition in the present study needed to be seriously considered as data were collected from Chinese school students, and the groups of students differed not only in their levels of expertise in the material to be learned, but also in their corresponding need to really learn the material for upcoming class examinations.

\section{The Present Study}

The findings of previous studies indicate that diagram construction and use is effective in learning, but that the diagram constructed must serve the requirements of the learning task and there needs to be sufficient cognitive resources available for both diagram construction and execution of the task. However, the question of how expertise (or lack of it) might affect these learning processes and outcomes had not been adequately examined. The question of how diagram construction promotes more successful learning also needs to be better understood. The present study therefore investigated these issues by testing the following hypotheses:

1. That diagram construction for learning would result in more units of ideas being encoded compared to summarizing;

2. That the amount of information students represent in diagrams and summaries would be related to their comprehension scores; 
3. That students who possess more knowledge about the topic would represent fewer units of ideas when learning (since they had previously learned the material already);

4. That students with less knowledge about the topic being learned would perceive higher levels of cognitive load when constructing diagrams.

The rationales for these hypotheses need to be explained. The first and second hypotheses arose from previously reported findings that generating diagrams was more effective than summarizing in facilitating text comprehension (Leopold \& Leutner, 2012). Although explanations have previously been proposed for the observed diagram strategy advantage (e.g., that it facilitates mental model building, and use of both visual and verbal channels of working memory), the question of how much information might get encoded when learners are constructing diagrams and writing summaries had not been considered. Because diagrams "can group together all information that is used together" and "support a large number of perceptual inferences" (Larkin \& Simon, 1987, p. 98), it is possible that diagrams simply enable learners to encode more of the crucial information from the texts they have to learn. Encoding more of the crucial information could in turn enhance learners' comprehension of the target text - as encoding would require attention to, and selection and representation of those particular units of information (e.g., Piolat, Olive, \& Kellogg, 2005). The first two hypotheses therefore tested whether diagram construction resulted in more units of information being encoded, and whether the 
amount of information represented in diagrams or summaries directly related to the students' comprehension test performance.

The third hypothesis might sound counterintuitive in that one might expect students with higher expertise to be able to include more relevant information in the diagrams and summaries they generate (compared to those with lower expertise). However, first, in the present study, "expertise" was relative and simply pertained to possession of prior experience in learning the subject matter of the target text material. Thus, the 8th-grade students were categorized as having "higher expertise" on account of their having experienced learning about the human blood circulation system in the previous academic year, which the 7th-grade students - categorized as having "lower expertise" - had not yet experienced. Second, as explained in the preceding section, previous research has shown that possession of prior knowledge in the material to be learned can lead to complacency in learning it (e.g., Wood \& Lynch, 2002), and that if students will not be examined in the material that they have been told to learn, their motivation for learning it will likely suffer (e.g., Chen et al., 2005). In the case of the 8th-grade participants in the present study, both of these potential impediments to learning were present as they were re-learning the information about the human blood circulation system, and they were no longer going to be tested in that subject matter in their real classes. Thus, the third hypothesis predicted that these 8th-grade students, who knew more about the topic, would represent fewer units of ideas when learning - for reasons of complacency and lower motivation. 
The fourth hypothesis was based on what cognitive load theory would predict (e.g., Kalyuga, 2011): that the amount of intrinsic load that a learner would experience would depend on the nature of the information he or she has to learn and his or her prior knowledge - or level of expertise - regarding that information. With lower levels of expertise (i.e., knowing very little or nothing about the matter to be learned), the experience of intrinsic load would increase (e.g., Van Merriënboer et al., 2006). As diagram construction was a learning strategy that some participants were tasked with in the present study, the fourth hypothesis simply predicted that those with less prior knowledge about the information to be learned - and to construct a diagram about would report experiencing higher cognitive load in undertaking this task.

\section{Method}

\subsection{Participants}

The participants were 34 8th-grade (approx. 14 years old; females =19) and 39 7th-grade students (approx. 13 years old; females =19) from a school in Beijing, China. The two classes that these students were drawn from were randomly selected from the classes at these grades available at the school. The classes were not streamed, so they contained students of mixed abilities. The students in the 8th-grade had previously received lessons about the human blood circulation system, which was the topic of the passage that they had to read and learn for the present study. Thus, for the purposes of this study, these students were designated as having "higher" expertise in the topic as they already possessed some domain specific knowledge about it as a 
consequence of having studied it in the previous academic year (cf. Sweller, 1988). In contrast, the 7th-grade students had not yet had those lessons, and were therefore considered as having "lower" expertise, on account of their not having studied the topic before and therefore presumably possessing little or no domain specific knowledge about it. In this experiment, students in both grades - and therefore, levels of expertise - were randomly assigned to either draw diagrams or to write summaries.

\subsection{Materials}

A four-page booklet was designed separately for the drawing diagram group and the writing summary group. The booklets were written in the Chinese language. The first page of the booklet was common to all participants, requiring them to provide demographic information, such as their gender, age, and year of study (grade level). Participants were not allowed to turn over pages of the booklet until the experimenter instructed them to do so (instruction to this effect was included at the bottom of each page). The second and third pages contained a total of four paragraphs (two paragraphs on each page) concerning the human blood circulation system (adapted and translated to Chinese from the material used in the Manalo \& Uesaka, 2012, study). Each paragraph was followed by a space designated for participants to either draw a diagram or write a summary of the key points (depending on group assignment). The instruction for what to do was given in writing. Translated to

English, it asked participants to either "Draw a diagram to represent the key points in this paragraph" or "Write a summary to represent the key points in this paragraph". After drawing the diagram or writing the summary for each paragraph, participants 
were required to provide a subjective rating of their cognitive load (How much mental effort did you have to put into drawing this diagram/writing this summary?). They were required to respond on a 7-point Likert-type scale where 1 = "extremely low" and 7 = "extremely high". Hence, in total, four cognitive load rating scales were included on pages 2 and 3 of the booklet. The last page of the booklet contained a comprehension test comprising of four questions about the human blood circulation system. The first three questions tested participants' understanding of details contained in the paragraphs and there was only one possible correct answer for each of them (e.g., Which part of the heart does the blood go to after it is oxygenated?). The last question required participants to make inferences from the information contained in the paragraphs, and there were multiple possible answers to it (i.e., A person is not getting enough oxygenated blood to the cells in many parts of his/her body. List three possible reasons for this person's problem). All questions required participants to write answers in the spaces provided following each question.

\subsection{Procedure}

Participant Assignment (5 minutes). Data collection was able to be conducted during students' regular class time as the topic dealt with in the study (human blood circulation) is part of the regular curriculum, and was either already covered in previous class sessions (for the 8th-graders) or to be covered in upcoming class sessions (for the 7th-graders). Numbered booklets labeled A (drawing diagram) or B (writing summary) were randomly distributed to participants who were seated in individual desks/chairs in a large classroom. Thus, group assignment depended on the 
booklet that each participant received. Participants were asked to provide answers to the demographic questions on the first page, and to follow the experimenter's instructions during the session.

Drawing Diagrams or Writing Summaries (20 minutes). After filling out all the required information on the first page of the booklet, the experimenter verbally instructed the participants to turn over to the second page, and to start reading each paragraph. This was followed by drawing a diagram or writing a summary, and then rating how much cognitive effort they had to use to draw or summarize the important points in each paragraph (but, as noted previously, these instructions were provided in writing in the booklet). Summary writing was selected as the learning strategy to compare with diagramming for two reasons: first, it was the comparison strategy used in the previously mentioned study by Leopold and Leutner (2012), and second, it appears to be the most common strategy used by students when learning from text that is, apart from other commonly used but clearly ineffective strategies such as simply reading over the text and underlining the text (e.g., Karpicke, Butler, \& Roediger, 2009; Slotte, Lonka, \& Lindblom-Ylänne, 2001). No instructions were provided to the students about how they should draw diagrams or write summaries, so this was left up to them to decide. Examples of diagrams and summaries produced by students are shown in Figure 1.

The participants were allowed 20 minutes to complete pages 2 and 3 of the booklet. Participants who completed the two pages more quickly were instructed to review the diagrams or summaries they had produced on those pages (i.e., the 
experimenter verbally announced that, "If you have already finished, please review

the work you have produced. Do not go to the fourth page until I tell you," and at the bottom of page 3, the words "Do not turn over until instructed" were written). After 20 minutes, the first three pages were detached and collected, and the participants were instructed to start on the comprehension test on the fourth page.

Comprehension Test (10 minutes).Ten minutes were allowed for participants to complete the comprehension test. Participants who finished earlier were asked to review their answers.

\subsection{Scoring}

The quality of the diagrams and summaries that the students produced were scored according to how many pre-determined units of ideas they contained. More specifically, there were 15 units of ideas that the authors considered important in the four paragraphs (see Table 1). During scoring, decisions were made on whether each of those units were represented or not in the diagrams and summaries the students produced. Each student therefore received a score out of 15 for the diagrams or summaries he or she produced. The first authors undertook all the data scoring, and the third author independently scored all of the 7th-grade students' diagrams and summaries. The kappa coefficient value for inter-rater agreement was found to be .64, which indicates "substantial" agreement (Landis \& Koch, 1977), so the authors deemed it unnecessary to calculate inter-rater agreement for the remainder of the data.

Each participant had four sub-scores for subjective ratings of cognitive load 
(one sub-score for each diagram or summary they produced). The average of those four sub-scores was calculated and used in the statistical analysis.

For the comprehension test, one point was given for each correct answer to questions 1 to 3 , and up to three points were given for question 4 depending on the number of correct reasons provided. Thus, each student received a score out of 6 .

\section{Results}

The data were analyzed by using a 2 (learning strategies: diagram vs. summary) x 2 (levels of expertise: low vs. high) MANOVA. Participants' scores on the number of idea units they represented, average cognitive load ratings, and the comprehension test were dependent variables.

\subsection{Effects on the Number of Idea Units Encoded}

The means and standard deviations of idea units participants represented, according to expertise and assigned strategy, are shown in Table 2.

[Insert Table 2 about here.]

The effect of learning strategy used was marginally significant, $F(1,69)=3.63$, MSe $=11.70, p=.061, \eta_{p^{2}}=.050$. This indicates a tendency for more idea units to be represented by students who drew diagrams compared to those who wrote summaries, which lends support to the first hypothesis. The effect of expertise was significant, $F(1,69)=15.88, \mathrm{MSe}=11.70, p<.001, \eta_{\mathrm{p}^{2}}=.187$. This result indicates that students with higher expertise (the 8th-graders) represented fewer idea units in their diagrams 
and summaries compared to the students with lower expertise (the 7th-graders). This result provides support to the third hypothesis.

The interaction between learning strategy used and expertise was not significant, $F(1,69)=2.37, \mathrm{MSe}=11.70, p=.128, \eta p^{2}=.033$.

\subsection{Effects on Perceived Cognitive Load}

The means and standard deviations of reported cognitive load experienced by participants, according to expertise and assigned strategy, are shown in Table 3.

[Insert Table 3 about here.]

The effect of expertise was significant, $F(1,69)=10.17$, MSe $=2.31, p=.002$, $\eta_{\mathrm{p}^{2}}=.128$. This finding indicates that participants with lower levels of expertise reported significantly higher levels of cognitive load than those with higher levels of expertise, which lends support to the fourth hypothesis. However, the main effect of learning strategy used was not significant, $F(1,69)=.92$, MSe $=2.31, p=.342, \eta_{\mathrm{p}^{2}}$ $=.013$, and the interaction between expertise and learning strategy used was also not significant, $F(1,69)=.04, \mathrm{MSe}=2.31, p=.846, \eta_{\mathrm{p}^{2}}=.001$.

As diagram use might be more effective for learning, participants who were in the diagram group were selected to further investigate the effect of expertise on the production of diagrams. Results revealed that the effect of expertise on cognitive load was significant again, $F(1,35)=4.71, \mathrm{MSe}=2.82, p=.037, \eta_{\mathrm{p}^{2}}=.119$, suggesting that although drawing diagrams leads to the representation of more idea units, it also 
imposes higher amounts of cognitive load particularly on participants who possess less knowledge or lower levels of expertiseon the material being learned. Incidentally, a similar result was obtained when the effect of expertise was examined in the summary group, $F(1,34)=5.78, \mathrm{MSe}=1.78, p=.022, \eta_{\mathrm{p}^{2}}=.145$. This result indicates that significantly higher amounts of cognitive load were experienced by participants with lower levels of expertise - even when summary writing was used as a strategy for learning.

\subsection{Results of Comprehension Test}

An important purpose of the comprehension test was to confirm that participants understood the content of the reading material provided about the human blood circulation system. An examination of the scores obtained by the grades 7 and 8 students showed that only around $10 \%$ of them obtained a score of 0 for this test, indicating that most participants understood at least some of the content and/or made adequate effort in answering the test.

The means and standard deviations of the participants' scores in the test, according to expertise and assigned strategy, are shown in Table 4.

[Insert Table 4 about here.]

The effect of expertise was found to be significant, $F(1,69)=6.95$, MSe $=2.99$, $p=.010, \eta_{\mathrm{p}^{2}}=.091$. Participants with lower levels of expertise scored higher in the test than those with higher levels of expertise (see Table 4). Considering this result together with the result about units of ideas represented suggests that the more idea 
units the students represented in their diagrams and summaries, the higher their comprehension test scores were, which confirms the second hypothesis. However, the main effect of learning strategy used was not significant, $F(1,69)=1.24$, MSe $=2.99$, $p=.269, \eta_{\mathrm{p}^{2}}=.018$, and the effect of the interaction between expertise and learning strategy used was also not significant, $F(1,69)=.14$, MSe $=2.99, p=.711, \eta_{\mathrm{p}^{2}}=.002$.

Correlational analysis was also conducted. This revealed that the amounts of idea units the students represented in diagrams and summaries were significantly and positively correlated with their comprehension scores: for diagrams, $\mathrm{r}=.479, p<.001$; for summaries, $\mathrm{r}=.406, p=.014$. This result provides further confirmation of the second hypothesis. In essence, the findings of this study suggest that the construction of diagrams in learning promotes the representation of more idea units from the material being learned. In turn, the more idea units students represent during the learning process, the better their comprehension of the material turns out to be.

\section{Discussion}

This experiment was designed to investigate the effect of expertise on the performance outcomes of using two different learning strategies: drawing diagrams and writing summaries. All four hypotheses were confirmed. The results suggest that drawing diagrams is more effective than writing summaries as it facilitates the representation of more of the important details from the material being learned, and the more of those details students represented during learning, the better they performed in the subsequent comprehension test. This finding is in line with previous 
studies attesting to the positive effects of using diagrams in learning situations (e.g., Leopold, \& Leutner, 2012; Leopold et al., 2015; Uesaka et al., 2007, 2010; Van Meter, 2001). However, it also provides a new explanation for why self-generated diagrams are effective in such situations.

As reported, learners with higher levels of expertise represented fewer idea units in the diagrams and summaries they generated. This finding may sound counterintuitive in that one might expect students with higher expertise to be able to include more relevant information in the diagrams and summaries they generate, compared to those with lower expertise. However, the 8th-grade students in this study had already previously studied the material about the human blood circulation system and they knew that they would no longer be tested on it in their regular class. It is therefore likely that they would have felt they knew the material already and would not have been highly motivated to try hard and study the material again (cf. Wood \& Lynch's, 2002, findings about the complacency that could come with re-learning). In contrast, the 7th-graders knew that the material dealt with an upcoming class topic they would need to really learn and sit tests for in the future, and so they were likely to have been more motivated in actually learning that material (cf. Chen, Warden, \& Chang's, 2005, comments about the motivational effects of tests on Chinese students). Thus, levels of expertise in this experimental context likely affected students' motivation to learn the materials they were presented, which in turn likely affected their efforts at representing idea units in the diagrams and summaries they produced. It is, of course, also possible that the 8th-grade students generated fewer idea units in 
the diagrams and summaries they produced because they had more mature schemas that enabled them to chunk some of the idea units together. However, even if they had more mature schemas, these students' likely lower levels of motivation/effort appears to have had a detrimental effect on their learning performance, as indicated by the significantly lower comprehension scores they obtained. Thus, a re-examination of this issue in future research - perhaps in conjunction with examining the effect of strategy use instruction on experience of cognitive load - would be useful.

The findings concerning cognitive load reported by participants during their learning tasks indicate that the more knowledgeable participants required lower amounts of cognitive effort compared to less knowledgeable participants in both diagram construction and summary writing. Constructing a suitable diagram requires students to translate verbal/textual information to a visual/image representation, which entails not only identification and selection of component parts that need to be included, but also decisions on the appropriate form that the diagram should take (Manalo \& Uesaka, 2012; Novick, Hurley, \& Francis, 1999). For more knowledgeable students, the intrinsic load of comprehending the material to learn is lower; therefore, they would have more cognitive resources available to use in executing those translational steps. In contrast, for more novice students, the intrinsic load of comprehending the material would be higher, and so they would have less cognitive resources available to deploy toward executing those translational steps. A similar advantage for more knowledgeable students would apply in summary writing, except the translational steps would involve selection and reduction of information, rather 
than a change to visual representation. These findings suggest that future studies ought to investigate how intrinsic load could be reduced, or how students' skills could be cultivated to enable more efficient translation between information representations.

Ideally, the lower level of intrinsic load could have made more cognitive resources available for the more knowledgeable participants to use in learning but, as noted above, this appeared not to have happened and instead they scored lower in both the number of idea units they represented and the comprehension test. Thus, as already suggested above, future investigations would need to control for or take motivation into consideration when examining the influence of expertise on student use of learning strategies. The authors are also aware that the different grades, and therefore ages, of participants in the high and low expertise groups were a potential confounding factor. Although higher age did not prove to be an advantage in this study, it would be useful in future studies to examine the issue of expertise in participants of the same age or grade level.

The finding that participants with lower levels of expertise on the material to be learned were able to represent more idea units during their learning as well as perform better in the subsequent test - despite the higher levels of cognitive load they reported - is promising. It provides support for the notion that effort and strategy use are more important determinants of learning performance than "ability" (or "expertise" in this case - cf. Ames \& Archer's, 1988, distinctions between types of achievement goals and associated learning behaviors). 


\subsection{Practical Implications}

The results of this study have two main educational practice implications. The first is that it would be beneficial to encourage students to make greater use of diagrams as a strategy for understanding information they are learning. The second is that it would be important to provide, especially for novice learners in a specific domain, extra instruction and/or guidance in diagram use for learning in order to free up more working memory resources to use for learning while constructing diagrams (cf. Manalo \& Uesaka, 2012, 2016). Based on the results of this study, we can assume that higher levels of cognitive load would be used in the process of translating textual information into appropriate diagrammatic representations during learning. This translation process would be cognitively demanding and effortful, more so for novices. Thus, it is essential that appropriate instruction be provided to guide this translation and reduce the levels of cognitive load entailed.

To conclude, the findings of the present study suggest that using diagrams is effective for identifying and encoding important information when learning, but that it would be helpful to provide instruction and/or guidance about diagram use/construction particularly to students who are novices in the topic area to reduce the potentially heavy cognitive load they experience when constructing diagrams. 


\section{Author Contributions}

The first author and the second author conceptualized and designed this research study, interpreted the results, and wrote and prepared this research manuscript. The first author and the third author conducted the data collection, coding, and analyses.

\section{Declaration of Competing Interests}

The authors declare no conflict of interest. 


\section{References}

Ainsworth, S., Prain, V., \& Tytler, R. (2011). Drawing to learn in science. Science, 333, 1096-1097.

Ames, C., \& Archer, J. (1988). Achievement goals in the classroom: Students' learning strategies and motivation processes. Journal of Educational Psychology, 80, 260-267.

Anzai, Y. (1991). Learning and use of representations for physics expertise. In K. A. Ericson, \& J. Smith (Eds.), Toword a general theory of expertise. Cambridge: Cambridge University Press.

Butcher, K. R. (2006). Learning from text with diagrams: Promoting mental model development and inference generation. Journal of Educational Psychology, 98, 182-197.

Chen, J. F., Warden, C. A., \& Chang, H.T. (2005). Motivators that do not motivate: The case of Chinese EFL learners and the influence of culture on motivation. TESOL Quarterly, 39, 609-633.

Cook, M. P. (2006). Visual representations in science education: The influence of prior knowledge and cognitive load theory on instructional design principles. Science Education, 90, 1073-1091.

Cox, R., \& Grawemeyer, B. (2003). The mental orgnization of external representations. In F. Schmalhofer, R. Young, \& G. Katz (Eds.), Proceedings 
of EuroCogSci03 (pp. 351-354). Mahwah, NJ: Lawrence Erlbaum.

De Bock, D., Verschaffel, L., Janssens, D., Van Dooren, W., \& Claes, K. (2003). Do realistic contexts and graphical representations always have a beneficial impact on students' performance? Negative evidence from a study on modelling non-linear geometry problems. Learning and Instruction, 13, 441-463.

Dufour-Janvier, B., Bednarz, N., \& Belanger, M. (1987). Pedagogical considerations concerning the problem of representation. In C. Janvier (Ed.), Problems of representation in the teaching and learning of mathematics (pp. 109-122). Hillsdale, NJ: Erlbaum.

Grawemeyer, B., \& Cox, R. (2004). The effect of knowledge-of-external-representations upon performance and representational choice in a database query task. Lecture Notes in Artificial Intelligence, 2980, $351-354$.

Grawemeyer, B., \& Cox, R. (2008). The effects of users' background diagram knowledge and task characteristics upon information display selection. Lecture Notes in Artificial intelligence, 5223, 321-334.

Hegarty, M., \& Kozhevnikov, M. (1999). Types of visual-spatial representations and mathematical problem solving. Journal of Educational Psychology, 91, 684-689. 
Kalyuga, S. (2011). Cognitive load theory: How many types of load does it really need? Educational Psychology Review, 23, 1-19.

Kalyuga, S., \& Sweller, J. (2005). Rapid dynamic assessment of expertise to improve the efficiency of adaptive e-learning. Educational Technology Research and Development, 53, 83-93.

Karpicke, J. D., Butler, A. C., \& Roediger, H. L. (2009). Metacognitive strategies in student learning: Do students practice retrieval when they study on their own? Memory, 17, 471-479.

Landis, J. R., \& Koch, G. G. (1977). The measurement of observer agreement for categorical data. Biometrics, 33, 159-174.

Larkin, J. H., \& Simon, H. A. (1987). Why a diagram is (sometimes) worth ten thousand words. Cognitive Science, 11, 65-99.

Leopold, C., Doerner, M., Leutner, D., \& Dutke, S. (2015). Effects of strategy instructions on learning from text and pictures. Instructional Science, 43, 345-364.

Leopold, C., \& Leutner, D. (2012). Science text comprehension: Drawing, main idea selection, and summarizing as learning strategies. Learning and Instruction, $22,16-26$.

Leutner, D., Leopold, C., \& Sumfleth, E. (2009). Cognitive load and science text comprehension: Effects of drawing and mentally imagining text content. 
Computers in Human Behavior, 25, 284-289.

Manalo, E., \& Uesaka, Y. (2012). Elucidating the mechanism of spontaneous diagram use in explanations: How cognitive processing of text and diagrammatic representations is influenced by individual and task-related factors. Lecture Notes in Artificial Intelligence, 7352, 35-50.

Manalo, E., \& Uesaka, Y. (2014). Students' spontaneous use of diagrams in written communication: Understanding variations according to purpose and cognitive cost entailed. Lecture Notes in Artificial Intelligence, 8578, 78-92.

Manalo, E., \& Uesaka, Y. (2016). Hint, instruction, and practice: The necessary components for promoting spontaneous diagram use in students' written work? Lecture Notes in Artificial Intelligence, 9781, 157-171.

Manalo, E., Uesaka, Y., Pérez-Kriz, S., Kato, M., \& Fukaya, T. (2013). Science and engineering students' use of diagrams during note taking versus explanation. Educational Studies, 39, 118-123.

Novick, L. R., Hurley, S. M., \& Francis, F. (1999). Evidence for abstract schematic knowledge of three spatial diagram representations. Memory \& Cognition, 27, 288-308.

Pellegrino, J. W., \& Hilton, M. L. (Eds.) (2012). Education for life and work: Developing transferable knowledge and skills in the 21st Century. Washington, DC: National Academies Press. 
Piolat, A., Olive, T., \& Kellogg, R. T. (2005). Cognitive effort during note taking. Applied Cognitive Psychology, 19, 291-312.

Schwamborn, A., Thillmann, H., Opfermann, M., \& Leutner, D. (2011). Cognitive load and instructionally supported learning with provided and learner-generated visualizations. Computers in Human Behavior, 27, 89-93.

Slotte, V., Lonka, K., \& Lindblom-Ylänne, S. (2001). Study strategy use in learning from text. Does gender make any difference? Instructional Science, 29, $255-272$.

Sweller, J. (1988). Cognitive load during problem solving: Effects on learning. Cognitive Science, 12, 257-285.

Sweller, J. (1994). Cognitive load theory, learning difficulty, and instructional design. Learning and Instruction, 4, 295-312.

Sweller, J. (2003). Evolution of human cognitive architecture. In B. Ross (Ed.), The psychology of learning and motivation, Vol. 43 (pp. 215-266). San Diego, CA: Academic Press.

Sweller, J., \& Chandler, P. (1994). Why some material is difficult to learn. Cognition and Instruction, 12, 185-233.

Sweller, J., Van Merrienboer, J. J., \& Paas, F. G. (1998). Cognitive architecture and instructional design. Educational Psychology Review, 10, 251-296.

Tindall-Ford, S., Chandler, P., \& Sweller, J. (1997). When two sensory modes are 
better than one. Journal of Experimental Psychology: Applied, 3, 257-287.

Uesaka, Y., Manalo, E., \& Ichikawa, S. (2007). What kinds of perceptions and daily learning behaviors promote students' use of diagrams in mathematics problem solving? Learning and Instruction, 17, 322-335.

Uesaka, Y., Manalo, E., \& Ichikawa, S. (2010). The effects of perception of efficacy and diagram construction skills on students' spontaneous use of diagrams when solving math word problems. Lecture Notes in Artificial Intelligence, $6170,197-211$.

Van Merriënboer, J. J., Kester, L., \& Paas, F. (2006). Teaching complex rather than simple tasks: Balancing intrinsic and germane load to enhance transfer of learning. Applied Cognitive Psychology, 20, 343-352.

Van Meter, P. (2001). Drawing construction as a strategy for learning from text. Journal of Educational Psychology, 93, 129-140.

Wood, S. L., \& Lynch, J. G. (2002). Prior knowledge and complacency in new product learning. Journal of Consumer Research, 29, 416-426. 
